Recherches en didactique des langues et des cultures

Les cahiers de l'Acedle

$15-3 \mid 2018$

Quelles médiations en didactique des langues et des cultures?

\title{
Anne Feunteun (2015) Des enfants et des langues à l'école. Les Éditions Didier, collection Langues \& didactique, Paris ; 310 pp.
}

Séverine Behra

\section{OpenEdition}

Journals

Édition électronique

URL : http://journals.openedition.org/rdlc/3807

DOI : $10.4000 /$ rdlc.3807

ISSN : 1958-5772

Éditeur

ACEDLE

Référence électronique

Séverine Behra, «Anne Feunteun (2015) Des enfants et des langues à l'école. Les Éditions Didier, collection Langues \& didactique, Paris; 310 pp. », Recherches en didactique des langues et des cultures [En ligne], 15-3 | 2018, mis en ligne le 01 septembre 2018, consulté le 20 avril 2019. URL : http:// journals.openedition.org/rdlc/3807

Ce document a été généré automatiquement le 20 avril 2019

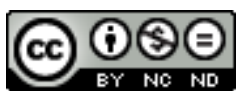

Recherches en didactique des langues et des cultures is licensed under a Creative Commons AttributionNonCommercial-NoDerivatives 4.0 International License 


\title{
Anne Feunteun (2015) Des enfants et des langues à l'école. Les Éditions Didier, collection Langues \& didactique, Paris ; 310 pp.
}

\author{
Séverine Behra
}

1 Vingt années de travail comme didacticienne, praticienne, formatrice et chercheure en France et en Europe sur la place des langues à l'école et leur enseignement aux enfants ont permis à Anne Feunteun de publier cet ouvrage en 2015. Elle y rassemble des connaissances référencées sur les travaux de recherche réalisés en DDLC et présente une synthèse diachronique des dynamiques de mise en œuvre de cet enseignement. Des enfants et des langues à l'école s'apparente à un état de l'art prenant appui sur celui de Charmian O'Neil intitulé Les enfants et l'enseignement des langues étrangères, paru vingt et un ans plus tôt. Feunteun part du constat, au moment où elle rédige son livre, que « la place des langues à l'école est toujours au cœur des débats à l'échelle de l'Europe » (p.6).

Le volume d'Anne Feunteun comporte 310 pages et s'organise en six chapitres assemblés par deux dans chaque partie. Le plan analytique retenu ici suit celui adopté précédemment par O'Neil (1993). Feunteun propose dans sa première partie une rétrospective et des perspectives historiques de l'enseignement des langues à l'école (pp. 13-95) ; la deuxième partie (pp. 97-182) s'attarde sur la « diversité linguistique et [la] transversalité des apprentissages au XXIe siècle "; enfin, le titre de la troisième partie est éponyme à celui de l'ouvrage et Feunteun mobilise ici, en fin d'ouvrage, les théories, concepts et notions de référence du domaine investi (pp. 183-276).

3 Ce livre s'adresse à un public concerné par les enfants et les langues, et, selon l'auteure,

tout particulièrement aux formateurs en didactique des langues, aux enseignants et aux futurs enseignants du premier et du second degré, aux étudiants et chercheurs en langues, en didactique des langues, en sciences du langage, en sciences de l'éducation, (...) [et] aux décideurs et responsables en matière de politiques éducatives linguistiques et culturelles (p.7). 
Cet ouvrage didactique comporte de nombreux encadrés qui illustrent le propos. Les titres des parties et chapitres ou les sous-titres référencés partent parfois de questions, comme dans un texte de vulgarisation qui répondrait à une foire aux questions. Par exemple, dès le chapitre 2 intitulé « Constantes, évolutions et questionnements » (p.58) il va s'agir de savoir s'il est important que les enfants apprennent les langues européennes (p.69); s'il y a un âge idéal (p.70); si les enseignants ont besoin d'une «maitrise linguistique ou [d'une] aisance pédagogique » (p.83). Comme le laisse entendre l'auteure dans le titre de ce chapitre, il est donc des questions récurrentes qui semblent traverser le temps à l'exemple de celle de l'« âge idéal » ou « âge optimum », comme on le disait vingt ans plus tôt. Cette question est traitée à deux reprises dans l'ouvrage, puisqu'elle se pose à propos des enfants dans le chapitre 5 (pp. 200-215).

5 Feunteun commence en retraçant l'histoire de l'évolution de l'enseignement des langues à l'école. Cette partie pourrait répondre aux questions de Duverger (2007) : « Alors où en est-on en France en 2006 [/2014] ? Comment peut-on problématiser la situation [de l'enseignement des langues à l'école élémentaire]?». Selon lui, pour répondre à ces questions, il est nécessaire de «reconsidérer l'ensemble de la problématique de l'enseignement des langues à l'école primaire, en relation avec une analyse politique sérieuse de l'état et de l'évolution des relations humaines dans le monde, en relation aussi avec les apports et les éclairages de chercheurs nationaux et étrangers aussi divers que sociologues et sociolinguistes, économistes et anthropologues, didacticiens et psychologues, en relation enfin avec les praticiens et les pédagogues » (Duverger, 2007). Feunteun s'inscrit dans cette démarche: son contexte d'observation et d'études est français et européen. Elle en présente les contours à l'échelle des événements mondiaux concomitants.

6 Toutefois, les repères temporels retenus par l'auteure sont parfois déstabilisants, en particulier dans le chapitre 1, parce qu'ils ne s'inscrivent pas toujours dans le continuum chronologique annoncé. Ainsi, le titre du chapitre 1 indique que la période étudiée est celle de 1992 à 1999 au cours de laquelle l'auteure souhaite observer "l'Europe en construction et les langues à l'école » (p.14 et suivantes). La première partie du chapitre nous invite à mieux comprendre la situation de l'enseignement des langues à partir de 1992 en se référant à l'histoire d'après-guerre et aux questionnements de H. Stern qui perdurent depuis les années 1960, à propos de l'enseignement des langues aux jeunes enfants en Europe et aux États-Unis, de son évaluation, de la formation des enseignants, etc. La deuxième partie du chapitre 1 s'arrête assez longuement sur l'année 1989, précédant donc à nouveau l'échelle temporelle annoncée dans le titre du chapitre (1992-1999). On se demande alors pourquoi le chapitre n'aurait pas pu couvrir la décennie de 1989 à 1999. L'anticipation d'une réponse peut apparaitre dans le fait que 1989 est décrite comme une année charnière pour l'enseignement des langues annonçant même une projection «dans le possible du plurilinguisme» (p.26) et annonçant surtout les prémisses des évolutions qui s'installent en 1992 pour en revenir aux considérations souhaitées de l'auteur en début du chapitre. Prenant appui sur une étude de 2001 intitulée L'Enseignement des langues étrangères en milieu scolaire en Europe et rédigée par l'unité européenne d'Eurydice, l'auteure confirme ainsi une «évolution majeure » entre 1974 et 1999 des langues enseignées en Europe. Elle complète ce diaporama de trois tableaux synoptiques de données issues de O’Neil (1993) pour illustrer la situation des langues dans les écoles de pays européens (pp. 28-29), avant de présenter plus en détail la situation de l'école française dans un tableau comparatif des textes officiels depuis 1989 
(pp. 34-35). Ce tableau marque trois des temps (1989-1992 / 1995-1998 / 1998-1999) de la valse des textes officiels. La description de chacune des orientations suivies reste simple et accessible. Elle est donnée à lire à l'aide d'une grille pour chacune des trois époques autour de mots clés communs : sigles/niveaux - choix de langues - objectifs - horaires enseignants - orientations didactiques - supports, qui nous ramènent à des pratiques scolaires évoquant des rubriques utilisées par les enseignants pour structurer leurs préparations. L'auteure ne développe pas plus avant la description de la réalité scolaire à l'œuvre à cette époque, tant elle est complexe, ayant peine à suivre les changements à répétition des règles dans l'imbroglio des textes officiels.

7 Feunteun termine ce chapitre par le rappel de l'importance du contexte politique, national ou régional qui guide les réflexions et les choix opérés. En effet, l'évolution de l'enseignement des langues en milieu institutionnel ne concerne pas uniquement le cas de l'apprentissage d'une langue étrangère nouvelle pour tous les élèves. Elle s'inscrit plus largement dans des spécificités nationales qui impliquent des "choix linguistiques" (p.40), en particulier dans le cas français avec l'accueil à l'école des enfants nouvellement arrivés (p.42) pour qui la langue de scolarisation est une langue nouvelle. S'appuyant à nouveau sur l'ouvrage d' O'Neil (1993) pour introduire cette sous-partie, l'auteure cite ici les travaux plus récents d'Auger (2010) pour s'attarder sur une description historique (pp. 42-45) des conditions d'accueil des enfants issus de l'immigration. L'observation en particulier des modalités de scolarisation de ces élèves met en lumière des questionnements méthodologiques et didactiques qui contribuent à la complexité de l'enseignement des langues. On se dit à ce moment-là que la question des langues à l'école dont souhaite traiter Feunteun, est plus complexe certainement et va au-delà des considérations de recherches centrées sur l'apprentissage d'une nouvelle langue-culture étrangère.

Or, le titre du second chapitre ramène le lecteur à considérer l'enseignement/ apprentissage des langues pour en observer les «Constantes, évolutions et questionnements» (p.58). Il laisse entendre que l'auteure va recentrer son débat en explicitant les finalités et objectifs attendus de cet enseignement. Inspirant moins d'instabilités temporelles, le titre de ce chapitre correspond, cette fois, à ce qu'il annonce. La comparaison des programmes européens pour l'apprentissage d'une langue étrangère à l'école révèle des choix nationaux communs en termes d'objectifs qui se définissent dans le cadre d'une démarche communicative, accordant une priorité à la communication orale. Tout cela sera expliqué plus longuement au cours de la deuxième partie de l'ouvrage. En revanche, il faut avouer que l'on ne s'attend pas dans la partie suivante de ce chapitre à ce que la question du choix des langues enseignées soit reliée à des résultats de sondages effectués à l'initiative du Conseil de l'Europe en 2001 et 2006. On regrette alors que ces résultats ne soient pas explicitement interprétés, par exemple en relation avec les politiques linguistiques des pays observés, pour mieux comprendre les choix opérés. Enfin, s'il reste une question récurrente pour terminer ce chapitre, c'est sans surprise celle de "l'âge idéal » pour commencer l'apprentissage d'une langue étrangère. On s'étonnera que la réponse apportée ici ne fasse pas état des apports de la recherche qui seront évoqués dans le chapitre cinq, pour se satisfaire du discours familial et de pourcentages sur le positionnement par pays des parents interrogés par sondages datés d'une dizaine d'années (2001 et 2006). L'opinion populaire fait ainsi de l'école élémentaire le terrain scolaire de prédilection pour l'introduction de l'apprentissage d'une langue étrangère, pour protéger les très jeunes enfants de toute surcharge cognitive dès l'école 
maternelle là où elle est institutionnalisée, ou pour ne pas attendre que l'enseignement secondaire s'en charge. D'autre part, les parents attendent que cet apprentissage garantisse une meilleure réussite professionnelle de leurs enfants (p.73). L'auteure note à juste titre que «l'opinion publique a beaucoup influencé la mise en place des langues à l'école » (p.76) pour expliquer l'intérêt de ces résultats. Cependant, on peut se demander comment être surpris que $91 \%$ des interrogés luxembourgeois (p.72) par exemple projettent cet apprentissage comme condition de meilleure réussite professionnelle, connaissant le contexte multilingue de ce pays ? Les écarts de pourcentage cités selon les pays sont difficilement comparables entre eux et ne tiennent compte ni de la situation de chaque pays, selon qu'il est multilingue ou non, ni de la situation de chaque répondant et de sa biographie langagière liées à un système de représentations et de croyances qui agit inévitablement sur le choix des réponses à des questions de sondage.

Le dernier point de ce second chapitre se centre en particulier sur les enseignants qui doivent s'adapter à l'enseignement des langues (pp. 77-95). Une présentation synthétique et schématisée des profils d'enseignants de l'école primaire en Europe conduit à la polémique bien connue en France de leur « niveau de compétence en langue » (p.81). Les professionnels de l'école primaire française ne sont pas recrutés en tant que spécialistesexperts d'une langue étrangère et l'auteure démontre ici comment les recherches dans le cadre d'études européennes, permettent de relativiser cette question. Il semble en effet qu'« une certaine fragilité linguistique des maitres peut être largement compensée par une grande aisance didactique» (p.86). Il resterait donc à en convaincre les futurs enseignants et les acteurs de la formation pour que dans les ESPÉ, l'essentiel de la formation initiale ne se concentre pas uniquement sur la maîtrise (tout au plus la pratique) idéalisée d'une langue étrangère. Ce point de vue fait aujourd'hui consensus pour défendre l'idée que la formation des enseignants de l'école primaire, telle qu'elle est actuellement proposée en Master MEEF (Métiers de l'Enseignement, de l'Éducation et de la Formation), pourrait davantage encore engager réellement ces futurs professionnels à « réfléchir à l'évolution de leurs représentations au contact de la différence culturelle, linguistique et pédagogique» (p.93). Il n'est toutefois pas si aisé en formation de faire émerger des croyances adossées aux propres biographies langagières des étudiants pour les faire évoluer. Ce travail s'avère cependant essentiel pour que la formation ne soit pas la reproduction d'une situation d'enseignement vécue et qu'elle contribue à un déplacement des pratiques scolaires, tant celles des enseignants que celles des élèves (Behra \& Macaire, 2017).

10 La deuxième partie de l'ouvrage, intitulée «Diversité linguistique et transversalité des apprentissages au XXIe siècle ", présente à nouveau des aspects des politiques éducatives européennes et décrit des choix didactiques engagés. Le chapitre 3 est essentiellement consacré au Cadre européen commun de référence pour les langues et aux portfolios à partir de 2001 (p.99). Après une genèse historique, ce cadre est interrogé comme marque d'une "évolution ou révolution didactique " (p.102). L'enseignement des langues à l'école désormais inscrit dans une démarche communicative s'engage également dans une perspective dite « actionnelle ». Le CECRL devient rapidement un outil de référence ; plus rapidement que les portfolios dont l'usage reste limité, voire très limité, même en 2018. Le portfolio est prévu pour accompagner l'apprenant dès ses débuts à l'école primaire et pour favoriser « une prise de conscience de ce que chacun fait ou pourrait faire pour apprendre les langues et interagir dans et avec ces langues » (p.109). L'apprenant se construit alors "une véritable culture d'apprentissage ", "affine » et "affirme [sa] 
capacité d'auto-évaluation et [sa] prise de conscience de son propre style pour apprendre ", tel que l'évoque Coste (2003), cité par l'auteure (p.109). Plus généralement, les portfolios « participent à la dynamique européenne d'ouverture du plurilinguisme et à la pluralité » (p.110). Cependant, comme s'il devait y avoir un revers de la médaille, les travaux de Beacco (2007), par exemple, montrent que le CECRL et les portfolios "permettent de prendre conscience des limites de l'approche communicative » (p.111) qui viserait essentiellement une pratique linguistique au détriment d'autres compétences de l'apprenant passées sous silence tant que les pratiques scolaires ne seront pas davantage centrées sur l'apprenant lui-même.

11 On regrette ici que l'état de l'art au sujet du CECRL et du portfolio n'aboutisse pas à des éléments critiques en complément de cette étude. L'auteure n'en explicite pas les limites. Elle n'évoque pas d'études liées à l'usage du portfolio à l'école, par exemple. Le portfolio existe dans sa version française sous forme d'un plateau de jeu qui permet de s'autoévaluer (p.110). Si son format ludique semble tout indiqué pour s'inscrire dans les usages de l'école primaire, on se demande alors pourquoi la génération des apprenants qui a connu la mise en place de l'enseignement d'une langue étrangère à l'école primaire et qui est actuellement en formation pour devenir de futurs enseignants de l'école primaire déclare, dans la grande majorité des cas, ne jamais avoir eu ou avoir su utiliser un portfolio. Comment expliquer le fait que l'outil prévu à cet effet n'accompagne pas systématiquement les débuts de l'apprentissage en langues ? Cette question, par exemple, n'est pas soulevée dans cet ouvrage.

Prenant appui sur le CECRL en faveur du développement pour le plurilinguisme et les compétences interculturelles, l'auteure revient à cet endroit de l'ouvrage avec des compléments sur les éléments retenus pour la définition de choix de politiques linguistiques à l'échelle européenne. Ces choix s'opèrent à la lecture de la situation propre à chaque contexte. Lorsque Feunteun s'arrête pour décrire la diversité des langues enseignées en Europe (pp. 114-116) à l'appui de chiffres clés européens, il en ressort que l'anglais, langue de grande diffusion, est la langue la plus apprise dans pratiquement tous les pays d'Europe et ce de plus en plus tôt (p.115), globalement aux alentours de six - sept ans. Toutefois, comme le note l'auteure, si l'on peut être satisfait que l'apprentissage d'une langue étrangère soit devenu une discipline obligatoire de l'école, on déplore encore le faible nombre d'heures consacré à cet apprentissage à l'école primaire (p.116). Et rien ne dit que l'anglais doit devenir une langue obligatoire à l'école. Il s'avère donc utile de poursuivre les réflexions et les recherches en DDLC qui permettront d'instaurer en contexte scolaire, le développement de compétences plurilingues chez les jeunes apprenants (pp. 116-120).

Dans la dernière partie de son chapitre, Feunteun s'intéresse aux «langues dans l'éducation, les langues pour l'éducation » (pp. 120-137). L'école est en effet le lieu où se rencontrent les langues de la maison, une langue de scolarisation et des langues apprises à l'école dans des proportions plus ou moins grandes pour chacune et contribuant ainsi à une forme d'hétérogénéité. Le propos de l'auteure décrit essentiellement le cas des langues de scolarisation, et ne considère pas les langues de l'environnement extrascolaire de l'apprenant. Tous documents d'appui, qu'il s'agisse du CECRL ou du Guide pour le développement et la mise en ceuvre de curriculums pour une éducation plurilingue et interculturelle, restent selon Feunteun «des propositions de curriculums à contextualiser" (pp.133-137) et à adapter aux conditions de développement de compétences plurilingues et à une certaine forme d'engagement dans la prise en charge 
de la diversité des langues à l'école. L'auteure ne contribue pas plus avant à la réflexion sur une possible mise en œuvre concrète, elle relaie l'exemple des propositions de Castellotti et Moore (2010) qui militent en faveur d'« un projet éducatif explicite » (p.137) au bénéfice de tous les apprenants que l'école accueille. Feunteun termine ce chapitre sur une note plutôt dramatique. Elle pose le constat que quelques pays et régions d'Europe s'organisent pour relever le défi qui consiste à tenir compte de l'enseignement des langues dans leurs réformes curriculaires, tandis que d'autres considèrent toujours que la didactique de la langue de l'école et la didactique d'une langue étrangère à l'école comme deux objets différents, qui se déclinent « côte à côte » (p.137) ; les langues de la migration semblent bien loin de toute considération, perçues comme « une autre question » (p.137), déconnectée pour ainsi dire de celle des langues au sens le plus large. Le cloisonnement des langues reste malheureusement un fait acté ici.

Le chapitre 4 décrit des exemples de mise en œuvre en contextes scolaires français plus particulièrement et européens d'« éducation transversale à la pluralité » (pp. 138-182). Ces exemples (pp.139-178), dont Feunteun revendique bien la non-exhaustivité, constituent un éventail de situations mises en œuvre depuis le début des années 2000 pour la plupart, localement ou régionalement, en appui de projets ou de programmes de recherche, voire soutenus par le biais d'associations, selon qu'il s'agira d'introduire des langues nouvelles à l'école ou de prendre en compte les langues présentes de l'immigration par exemple, les deux cas les plus détaillés parmi ces exemples. On s'arrêtera ici sur le dernier point du chapitre «Langues des enfants et langues à l'école » (pp. 168-182). Comme le dit Feunteun, pour l'enseignant, « l'école peut être à la fois un lieu de normalisation, de transmission, de hiérarchisation des langues et de leurs valeurs et/ou de valorisation, au sein même de ses enseignements »(p.168). L'école est traditionnellement organisée autour de pratiques compartimentées qui ne permettent pas aisément, ni suffisamment, d'articulation, ou qui, quand elles existent, ne sont pas toujours rendues explicitement accessibles à l'ensemble des élèves. En fait, Feunteun évoque ici une organisation scolaire qui s'inscrit dans une vision cumulative des langues, tel que le suggère la dénomination d'un cursus scolaire d'écolier qui s'engage dans l'apprentissage d'une langue vivante étrangère $\mathrm{n}^{\circ} 1$, puis une « LV2 » et parfois même une "LV3", sans se demander si la langue de scolarisation ne serait pas déjà une «langue quasi étrangère " pour certains élèves. L'école a voulu « ranger ", classer, organiser, alors que, comme le redit Feunteun à la suite de Simon (2006), «la problématique didactique globale de langue de l'école / langue nouvelle / langue(s) familiale(s) / éveil aux langues et culturelles » demande à être repensée (p.171). L'auteure propose alors « d'adopter une démarche transversale réflexive où chaque élément de ce qui est enseigné et découvert dans la classe s'articule, s'interroge et se renforce réciproquement»(p.172). Cette démarche se réalisera dans l'articulation de projets variés. Il s'agit, selon l'auteure, de proposer « une mise en œuvre pédagogique à la fois holistique, expérientielle, réflexive et écologique des apprentissages à l'école » (p.172). L'auteure se trouve elle-même impliquée dans des projets de démarche didactique intégrée proposés à des élèves de cycle 3 (Feunteun, 2007, 2014) visant à articuler la langue de scolarisation, la langue étrangère apprise à l'école et des activités d'éveil aux langues-cultures issues des ressources de la classe (p.173). L'auteure ne dresse pas une liste exhaustive de tous les projets qui peuvent être proposés. Elle aurait pu faire référence ici aux projets du corpus présenté aux pages 139 à 178, évoqués supra, pour l'enseignement des langues à l'école. Tous les projets créés s'adaptent aux contextes et aux profils des apprenants (p.182). 
15 La dernière partie de l'ouvrage d'Anne Feunteun commence par un chapitre consacré aux enfants (p. 184); ces enfants dont on attendait qu'ils soient au cœur de cet ouvrage, prenant la première place dans le titre du livre. L'enfant est interrogé en tant qu'apprenant de langues dans ce chapitre qui rappelle tant les théories d'acquisition que celles d'apprentissage et les stratégies développées par les apprenants. Ce chapitre s'arrête de nouveau sur la question de l' "âge idéal » pour commencer l'apprentissage d'une langue étrangère (pp. 199-222), non plus pour recueillir l'avis des parents (cf supra, chapitre 2), mais pour présenter les situations qui selon l'auteure aident à mieux percevoir dans quel cas un apprentissage précoce peut être un avantage, partant du principe que "les chercheurs ne sont toujours pas parvenus à un consensus clair » (p.200) sur cette question. Dans l'opposition entre avantages et inconvénients d'un apprentissage précoce d'une langue à l'école, le débat ne tient probablement pas seulement à la notion d'âge. Selon l'auteure, «la question des effets immédiats et des effets à long terme d'un apprentissage précoce d'une langue nouvelle demeure la question clé pour l'ensemble des acteurs en matière d'éducation» (p.201). De manière plus générale encore, la question essentielle qui reste posée est celle de l'objectif de l'apprentissage des langues à l'école primaire (p. 207).

16 À la lecture du titre du dernier chapitre de l'ouvrage «enseigner une/des langues nouvelles à l'école » (p.223), le lecteur s'attend à ce que ce chapitre soit davantage centré sur les enseignants. Or, les vingt-cinq premières pages de ce chapitre concernent à nouveau l'apprentissage et l'appropriation des langues par l'enfant (pp. 225-252) pour présenter une synthèse des recherches en psychologie cognitive et en sociolinguiste. L'enseignant n'y apparait qu'en filigrane peu avant la fin du chapitre, lorsque sont évoquées les questions d'évaluation (pp. 252-259). Ces dernières demeurent souvent problématiques pour les enseignants et nombre d'entre eux se sentent démunis (p.252) pour percevoir clairement le niveau atteint de leurs apprenants. L'auteure pose le constat qu'en France comme dans la plupart des pays, l'enseignement des langues peine à «se démarquer de l'exigence native normée» (p.256). Or, selon Feunteun, «il s'agit de dédramatiser l'enseignement et l'évaluation d'une langue nouvelle à l'école en adoptant un nouveau regard sur une évaluation qui se veut positive et encourageante » (p.253); ce qui ne peut être compris qu'à condition de clarifier les objectifs de cet apprentissage. Feunteun se demande en fin de chapitre s'il s'agit d' "apprendre à communiquer ou communiquer pour apprendre? » (p.259). Cette question revient donc à nouveau à poser la question de l'objectif de cet apprentissage qui pourrait donc être résolue avant la fin de l'ouvrage.

17 Il s'avère que l'approche communicative dans laquelle s'inscrivent les enseignements de langues n'explicite pas les objectifs d'apprentissage des langues (p.264). Cette approche a certes largement contribué à mieux percevoir les besoins de l'apprenant, et surtout à mieux comprendre ce qu'est une situation d'échanges. Cela ne suffit pas cependant à garantir que l'apprenant devienne systématiquement équipé de la compétence de communication, surtout si on ne peut s'assurer qu'il possède une "compétence d'autonomie stratégique » pour lui permettre par exemple, de s'autoévaluer, de réfléchir à la façon dont il apprend les langues (p.265). L'enjeu ne consiste pas seulement à percevoir les besoins de l'apprenant (cf. supra), mais plutôt à connaitre l'apprenant luimême, sa biographie langagière (Feunteun n'utilise que le terme de «biographie » dans le sous-titre de sa partie) et ses représentations quant aux objectifs de son engagement dans les apprentissages de langues proposés. L'auteure termine sur cette préconisation: «la 
langue de scolarisation et les langues nouvelles devraient s'appréhender de manière interdépendante au moyen de contenus adaptés et en fonction de situations et d'objectifs précis » (p.279). Et ses derniers mots rejoignent ceux d'o'Neil (1993) qu'elle cite en clôture de propos sur ce domaine où "l'innovation et l'imagination trouvent encore leur place » (p.279).

En conclusion, cet ouvrage résume vingt années de travail de l'auteure sur une soixantaine d'années de politiques linguistiques éducatives en Europe à l'appui de références scientifiques et statistiques. Il expose des observations de mises en œuvre d'enseignement des langues à l'école, et des réflexions qui, comme le dit Feunteun, « sans relâche, ont ébranlé des systèmes, interrogé des chercheurs, des institutionnels, des décideurs, des concepteurs, des formateurs et des enseignants»(p.277). Néanmoins, toutes les situations de mise en œuvre ne sont pas comparables, les contextes ne l'étant pas par ailleurs. L'auteure ne cherche pas à montrer, par exemple, comment l'expérimentation réussie d'un projet dans un pays pourrait être transposée pour d'autre, et à quelles conditions.

L'auteure précise que cet ouvrage ne prône, ni ne cherche à défendre une "bonne manière » d'enseigner qui serait "pertinente partout et pour tous» (p.277). Il est toutefois regrettable que l'auteure n'engage pas davantage de critique, positive ou négative, de certains concepts et outils qu'elle décrit dans cette synthèse. Bien que structuré minutieusement en apparence dans le déroulement du plan suivi, l'exposé n'est pas toujours simple à lire. Les renvois sont nombreux tant sur un axe temporel qu'entre les chapitres du livre. En outre, on peut regretter que l'adéquation entre les titres des chapitres ou parties et leur contenu ne soit pas systématiquement rendue plus clairement perceptible, ainsi qu'un manque d'articulation entre les sous-parties d'un chapitre qui prend alors plus l'allure d'un catalogue d'idées sur la question de l'enseignement des langues et non d'une réelle problématique que l'auteure empoignerait à bras le corps pour en dégager des orientations de recherche, des pistes d'action, des questionnements nouveaux dans le cadre d'un rebrassage des notions. Le lecteur regrette enfin qu'on s'y reprenne à deux fois pour décrire les politiques linguistiques, ou pour répondre à la question de "l'âge idéal», par exemple. Il ne s'agit certes pas de redites, mais de différentes phases d'analyse présentées au lecteur sans aboutir réellement à un éclairage nouveau sur la question. Les renvois contextuels historiques et géopolitiques sont agréables parce qu'ils peuvent évoquer des souvenirs, des images de journaux d'informations, et à la fois ils retardent trop souvent la réflexion attendue. L'ouvrage est descriptif et les notions, concepts et outils présentés ne sont pas (ré)interrogés ou critiqués pour s'engager vers des perspectives futures.

\section{BIBLIOGRAPHIE}

Behra, S. \& Macaire, D. (2017) : « Pisteurs de croyances : pratiques et formation initiale du premier degré », Études en Didactique des Langues, 28, "Stéréotypes, croyances, représentations », Toulouse, 115-136. 
Duverger, J. (2007) : « École élémentaire et enseignement des langues », Tréma [En ligne], 28 | 2007, mis en ligne le 13 janvier 2010, consulté le 25 avril 2018. URL : http:// journals.openedition.org/trema/259; DOI : 10.4000/trema.259.

O’Neil, C. (1993) : Les enfants et l'enseignement des langues étrangères, LAL-CREDIF, Hatier, Paris.

INDEX

Thèmes : Comptes rendus

\section{AUTEUR}

\section{SÉVERINE BEHRA}

Séverine Behra est maître de conférences à l'Université de Lorraine / ESPÉ de l'Académie de Nancy-Metz. Membre du laboratoire ATILF (UMR 7118), équipe Didactique des langues et sociolinguistique, axe CELMI (Cultures Éducatives et Langues en Milieu Institutionnel). Severine.behra[at]univ-lorraine.fr 